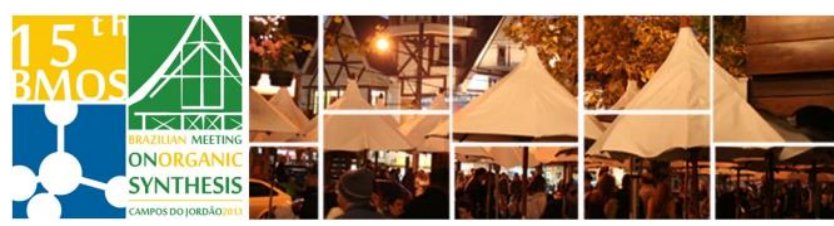

\title{
Directed Magnesiation of Haloaromatic Oxazolines using the mixed lithium/magnesium base TMPMgCl.LiCl
}

\author{
Batista, J.H.C., Santos, F.M., Silva, S.C., Oliveira, A.R.M., Clososki, G.C \\ Faculty of Pharmaceutical Sciences of Ribeirão Preto, University of São Paulo \\ *e-mail corresponding author: joaohenrique08@yahoo.com.br
}

Keywords: Fuctionalization, oxazolines, TMPMgCl.LiCl

\section{INTRODUCTION}

The metallation of aromatics is a convenient approach to the functionalization of unsaturated scaffolds ${ }^{1}$. Polyfunctional aryl halides are of high importance as agrochemicals, pharmaceuticals and building blocks ${ }^{2}$. Recently, mixed lithium/magnesium amides such as TMPMgCl. LiCl and $\mathrm{TMP}_{2} \mathrm{Mg} .2 \mathrm{LiCl}$ have proven to be interesting bases for functionalization of arenes under mild conditions ${ }^{3}$. In this work we wish to report the use of magnesium amides for the magnesiation of several haloaromatics oxazolines and subsequent reactions with electrophiles.

\section{RESULTS AND DISCUSSION}

The oxazolines were prepared by condensation of aldehydes with aminoalcohol, providing the products with yields ranging from 75 to $90 \%$ (Scheme 1).

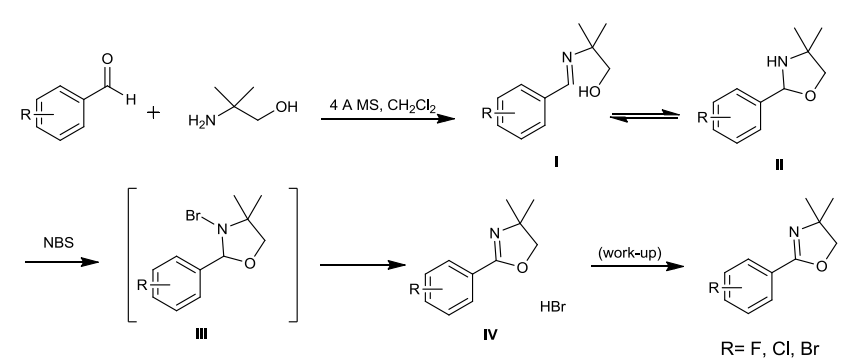

Scheme 1: Preparation of oxazolines.

The mixed $\mathrm{Li} / \mathrm{Mg}$ base was obtained through the direct reaction of 2,2,6,6-tetramethylpiperidine (TMPH) with $i$-PrMgCl.LiCl (Scheme 2).

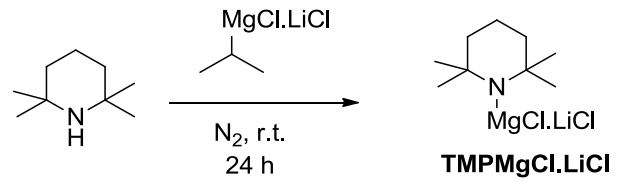

Sheme 2: Preparation of TMPMgCl.LiCl

The magnesiation of haloaromatics oxazolines with TMPMgCl.LiCl was achieved within $2 \mathrm{~h}$ at room temperature. Further reaction several electrophiles led to the expected functionalized oxazolines in good yields (Scheme 3, Table 1).

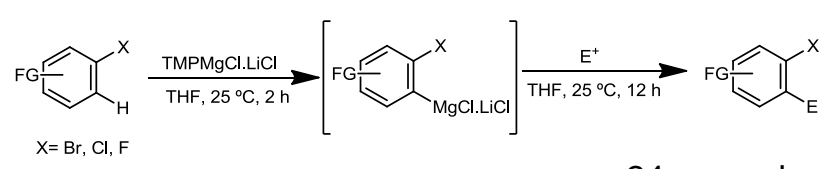

24 examples

Scheme 3: Magnesiation of oxazolines

Table 1. Products obtained after directed magnesiation of oxazolines

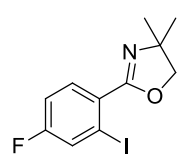

$85 \%[a]$

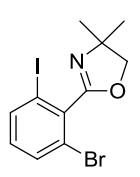

$55 \%$ [a]
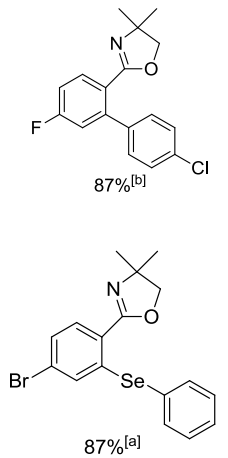
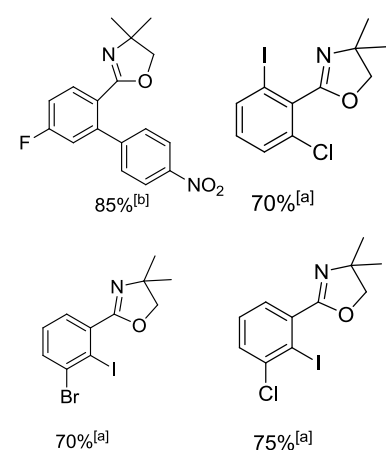

[a] Yield of isolated, analytically pure product.

[b] A transmetalation with $\mathrm{ZnCl}_{2}$ (1.1 equiv.) and $\mathrm{Pd}$-catalyzed cross-coupling using $2 \mathrm{~mol} \% \mathrm{Pd}(\mathrm{dba})_{2} 4 \mathrm{~mol} \%$ and $\mathrm{tfp}$ were performed.

\section{CONCLUSION}

The metallation of haloaromatic oxazolines using TMPMgCl.LiCl was successfully achieved under mild conditions. The resulting Grignard reagents can be combined with a large number of electrophiles to provide highly functionalized oxazolines in good yields.

ACKNOWLEDGEMENTS
FAPESP, CNPq, CAPES.
REFERENCES

${ }^{1}$ a) N. Chatani, Directed Metallation, in: Topics in Organometallic
Chemistry, Springer, Berlim, 2007. b) R.E. Mulvey, F. Mongin, M.
Uchiyama, Y. Kondo, Angew. Chem. 2007, 119, 3876.
2 K.C. Nicolaou, T. Montagnon, Molecules that Changed the
World: A Brief History of the Art and Science of Synthesis and its
Impact on Society, Wiley-VCH, Weinheim, 2008.
${ }^{3}$ T. Kunz, P. Knochel, Angew. Chem. 2012, 124, 1994; Angew.
Chem. Int. Ed. 2012, 51, 1958.

